Revue de droit comparé du travail et de la sécurité sociale

2| 2019

L'ubérisation du travail

\title{
L'ubérisation du travail dans la jurisprudence brésilienne
}

Sidnei Machado

\section{(2) OpenEdition}

1 Journals

Édition électronique

URL : https://journals.openedition.org/rdctss/1559

DOI : 10.4000/rdctss. 1559

ISSN : 2262-9815

Éditeur

Centre de droit comparé du travail et de la sécurité sociale

Édition imprimée

Date de publication : 1 juin 2019

Pagination : 76-81

ISSN : 2117-4350

Référence électronique

Sidnei Machado, "L'ubérisation du travail dans la jurisprudence brésilienne », Revue de droit comparé du travail et de la sécurité sociale [En ligne], 2 | 2019, mis en ligne le 01 novembre 2021, consulté le 11 novembre 2021. URL : http://journals.openedition.org/rdctss/1559; DOI : https://doi.org/10.4000/ rdctss. 1559

\section{(c) (i) (9)}

Revue de droit comparé du travail et de la sécurité sociale est mise à disposition selon les termes de la Licence Creative Commons Attribution - Pas d'Utilisation Commerciale - Pas de Modification 4.0 International. 


\section{L'UBÉRISATION DU TRAVAILDANS LA JURISPRUDENCE BRÉSILIENNE}
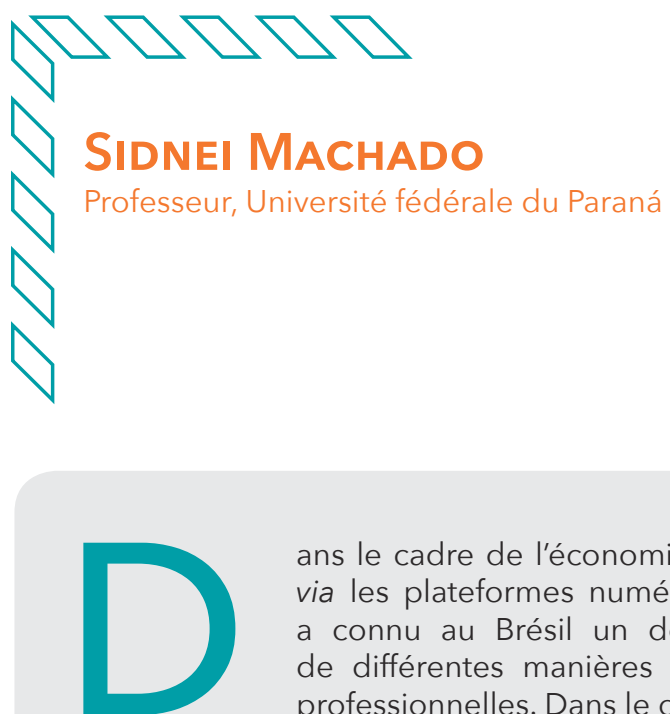

ans le cadre de l'économie de partage, le travail à la demande, via les plateformes numériques de transport et de distribution a connu au Brésil un développement rapide qui a impacté de différentes manières le marché du travail et les relations professionnelles. Dans le cas spécifique du Brésil, ces innovations technologiques suscitent une préoccupation particulière : elles peuvent en effet être potentiellement liées à des formes de précarité - comme celles qui ont caractérisé le marché du travail dans le passé - et ouvrir ainsi la voie à l'émergence de formes nouvelles et moins protégées de flexibilité dans les relations de travail. En l'absence d'une réglementation suffisante, le principal défi consiste, en ce qui concerne ces nouveaux emplois issus de la numérisation du travail, à déterminer la qualification des relations contractuelles.

Au Brésil, les chauffeurs des plateformes numériques, telles qu'Uber et Cabify, sont explicitement considérés comme des travailleurs indépendants, liés par des contrats de prestation de service ou entretenant tout simplement des relations de travail informelles. Les services de transports privés brésiliens utilisant des plateformes numériques ont été réglementés en 2018 par la Loi n 13.460/2018, qui aborde les questions fiscales et les obligations envers l'Etat. Si cette loi n'a pas eu pour objectif de réglementer les relations de travail, la norme brésilienne permet toutefois aux chauffeurs de réclamer la sécurité sociale en tant que travailleurs indépendants, ce qui suggère clairement l'absence d'une relation salariée. L'adhésion individuelle à la sécurité sociale correspond généralement au statut d'entrepreneur (Micro Empreendedor Individual MEI - Loi complémentaire 128 de 2006), qui donne accès à certaines prestations sociales telles que la pension de retraite, mais pas aux autres droits propres aux travailleurs salariés (négociation collective, salaire minimum, durée du travail maximale, etc.). 
Du point de vue institutionnel, un ensemble de normes régissant les relations de travail a été adopté au cours des dix dernières années, afin d'assimiler ces travailleurs à des indépendants. On peut citer, par exemple, l'institutionnalisation de l'entreprise individuelle à responsabilité limitée (EIRELIS, art. 980-A du Code civil, introduite par la Loi $n^{\circ} 12.441$ de 2011) et celle du transporteur de frêt indépendant (Lois $n^{\circ} 11.442$ de 2007 et n¹3.103 de 2015). Un changement plus net de paradigme s'est produit lors de la réforme de la législation du travail de 2017 (Lois $n^{\circ} 113.429$ et $n^{\circ 13.467)}$ ) avec des mesures fortes de déréglementation permettant à une entreprise de sous-traiter toute forme d'activité, y compris celles qui ne sont pas ponctuelles. Un contrat de travail intermittent fut également institué, parmi d'autres mesures contribuant à fragmenter les relations de travail et à compliquer la détermination du statut de la main-d'œuvre dans les domaines hybrides du monde du travail.

C'est dans ce contexte institutionnel complexe, extrêmement dommageable aux fonctions de la réglementation des relations de travail, que des tribunaux du travail brésiliens ont abordé les questions relatives aux différentes formes de contrats de travail, résultant des subtilités du travail sur les plateformes numériques, un travail qui se révèle aussi novateur que perturbateur. Quelle relation lie la plateforme, le travailleur et le consommateur? La relation entre l'individu et la plateforme est-elle une relation de travail typique ou s'efforce-t-on de dissimuler la relation de travail réelle?

Face à une réalité complexe, illustrée par le développement du travail sur les plateformes numériques, la jurisprudence des tribunaux du travail a abouti à un ensemble de décisions, issues de conflits individuels, portant sur le problème de la qualification juridique de la relation contractuelle engendrée par l'ubérisation du travail. Les décisions jurisprudentielles pertinentes sont encore peu nombreuses et il n'existe toujours pas de décision susceptible d'être considérée comme un précédent.

Les décisions judiciaires concernant Uber - une cinquantaine d'affaires jugées par les instances spécialisées en droit du travail jusqu'en mars 2019 - sont plus nombreuses que les conflits relatifs à d'autres plateformes numériques. Même si cela ne constitue pas, à ce jour, une jurisprudence consolidée, ces décisions contribuent au débat dans la sphère publique. Sur la base de ces décisions rendues par les tribunaux brésiliens du travail depuis 2017, en particulier dans les cas concernant la nature des relations de travail entre Uber et ses chauffeurs, il est possible d'identifier les éléments les plus pertinents (III), systématiquement utilisés par les tribunaux du travail brésiliens dans la caractérisation des relations de travail, à savoir la catégorie de salarié (I) et celle d'employeur (II).

\section{I - LE CONCEPT DE SALARIÉ}

Une première décision judiciaire a été rendue le 13 février 2017, suite à un litige individuel impliquant un chauffeur et l'entreprise Uber (Uber do Brasil, Tecnologia LTDA., Uber International B.V. et Uber International Holding B.V.). Le chauffeur, un travailleur de 39 ans qui avait fourni ses services à Uber pendant onze mois en 2015, alléguait l'existence d'une relation de travail subordonné et exigeait donc la reconnaissance de son statut de salarié de la société. L'argumentation adoptée par Uber pour sa défense, essentiellement basée sur son modèle d'affaires, affirmait l'existence d'une relation de partenariat, excluant ainsi le statut de salarié. Selon la société, (i) Uber se définit comme une entreprise de technologie et non comme une entreprise de transport, (ii) le chauffeur partenaire dispose ainsi de la liberté de choisir de se connecter, d'accepter ou de refuser une course, (iii) il n'existe aucune exclusivité dans les prestations fournies à Uber par le chauffeur. 
La décision rendue par un juge du travail en première instance a reconnu l'existence d'une relation de travail entre le chauffeur et Uber, une décision qui eut un fort écho dans les médias puisque ce tribunal fut le premier du pays à se prononcer en faveur de la reconnaissance du statut de salarié pour un chauffeur d'Uber. Pour justifier l'existence de la relation de travail, le juge a estimé que toutes les conditions imposées par le droit du travail brésilien pour constater l'existence d'une relation employeur-salarié étaient réunies : (i) la relation entre la société et la personne physique, (ii) le caractère personnel de la relation (seul le chauffeur inscrit peut conduire le véhicule), (iii) le caractère pécuniaire (la rémunération est prise en charge par la société qui en fixe les montants et les conditions), (iv) l'absence de caractère ponctuel ou de caractère habituel (le service n'est pas fourni sporadiquement), (v) la subordination (les chauffeurs sont soumis à un code de conduite défini par Uber).

Pour justifier juridiquement l'existence dulien de subordination, le juge a estimé que les chauffeurs devaient non seulement suivre des règles strictes pour pouvoir continuer à travailler, mais ils devaient également obtenir de bonnes évaluations pour rester " partenaires » d'Uber, avec une autorisation d'accès à la plateforme. La décision concluait que le chauffeur « était soumis à des instructions sur la manière de fournir les services et devait se plier à des formes de contrôle continu. En outre, il était passible de sanctions disciplinaires en cas de comportement répété jugé inapproprié ou en cas de violation du code de conduite défini par le défendeur ${ }^{1}$ ». En ce qui concerne les éléments qui ont motivé la décision sur le pouvoir de contrôle de la plateforme, le juge a affirmé que : "à l'ère de l'efficacité cybernétique, il est nécessaire de porter attention au pouvoir de l'employeur, qui s'exerce également par l'intermédiaire de systèmes programmés, d'algorithmes et de réseaux ${ }^{2} »$.

Cependant, cette décision judiciaire a été infirmée en deuxième instance le 23 mai 2017, par le tribunal régional du travail de Minas Gerais (TRT-MG) qui a donné raison à Uber, considérant que les conditions légales caractérisant une relation employeur-salarié n'étaient pas remplies. Pour justifier sa décision, le tribunal de deuxième instance a estimé qu'Uber était une plateforme numérique ayant pour objectif de connecter les chauffeurs et les utilisateurs enregistrés, que les travailleurs étaient libres de décider des heures de travail, et qu'il n'existait pas d'obligation personnelle. La Cour a donc conclu à l'absence de lien de subordination juridique " parce que le défendeur est une plateforme numérique qui vise à connecter les chauffeurs enregistrés aux usagers des transports ${ }^{3}$ ».

Les débats autour de ces décisions de justice ont pour point commun de se focaliser sur la qualification adéquate des paramètres qui délimitent le concept de salarié dans le cadre du travail via des plateformes numériques. En ce sens, la controverse est centrée sur le concept de lien de subordination, facteur unique et déterminant de la relation de travail. Dans la décision qui a reconnu l'existence d'une relation employeur-salarié, le juge soumet le travail via des plateformes au modèle classique du lien de subordination pour retenir la thèse du contrôle permanent et de la subordination. En revanche, dans la décision révisée,

1 Brésil, $33^{\mathrm{a}}$ Vara do Trabalho do Tribunal Regional do Trabalho da $3^{\mathrm{a}}$ Região, procédure $\mathrm{n}^{\circ} 0011359-$ 34.2016.5.03.0112, p. 21.

2 Brésil, Tribunal Regional do Trabalho da $3^{\text {a }}$ Região, procédureo n 0011359-34.2016.5.03.0112, procédure n0011359-34.2016.5.03.0112, p. 21.

3 Brésil, Tribunal Regional do Trabalho da $3^{\text {a }}$ Região, procédureo n 0011359-34.2016.5.03.0112, p. 17. 
les éléments relatifs à la possibilité de fixer le temps de travail, d'accepter ou de refuser un travail, motivent le rejet de la subordination juridique.

\section{II - LE CONCEPT D'EMPLOYEUR}

Le tribunal régional du travail de São Paulo a adopté des points de vue divergents dans ses analyses et arguments concernant d'autres décisions judiciaires relatives à Uber. Ainsi, le tribunal de São Paulo a reconnu le statut de salarié d'un chauffeur d'Uber dans une décision rendue en août 2018, estimant que le chauffeur ne disposait pas d'une autonomie réelle face à l'obligation de respecter le code de conduite défini par l'entreprise. La décision reposait sur le "caractère fallacieux de l'argument utilisé, puisqu'il existe un contrôle du service fourni par le chauffeur, appelé 'partenaire'. S'il ne s'agissait que d'un instrument électronique, les défendeurs ne suggèreraient certainement pas le prix du service de transport fourni, ni le pourcentage destiné au chauffeur ». Afin de démontrer l'existence d'un lien de subordination juridique, le tribunal a estimé que « les entreprises utilisent des mécanismes indirects pour obtenir du chauffeur la disponibilité maximale souhaitée et pour l'affecter aux utilisateurs qu'elles servent ${ }^{4} »$.

La décision a également fait valoir que l'évaluation des performances par les clients qui décident de l'avenir du conducteur sur la plateforme constitue un mécanisme de contrôle. S'agissant de l'application de la notion traditionnelle de subordination, le juge a considéré que : " la relation existant entre les défendeurs et les chauffeurs qui travaillent pour eux ne correspond pas au modèle classique de la subordination et, selon le cas analysé, il pourrait ne pas y avoir de reconnaissance du statut de salarié, notamment dans les cas où la prestation de services se révélerait être ponctuelle ${ }^{5}$ ".

Dans une précédente décision, rendue par le même tribunal du travail de São Paulo le 14 décembre 2017, l'existence du statut de salarié n'avait pas été reconnue et le tribunal avait, à l'inverse, conclu que la relation entre la plateforme et le chauffeur ne répondait pas aux critères de travail régulier, personnel et de subordination. La décision précisait ainsi : "Le système Uber est en réalité une plateforme technologique facilitant le contact entre un propriétaire de véhicule enregistré et un client. [...] Les moyens technologiques servent à améliorer et à faciliter le mode de vie des gens. La prestation étant fournie au nom de la société, laquelle est également responsable des irrégularités pouvant être commises par le chauffeur, il appartient à la société d'établir des règles autour de la prestation des services, règles qui ne doivent pas être confondues avec le pouvoir de direction d'un employeur. A partir du moment où le chauffeur s'enregistre sur la plateforme d'Uber, il adhère à plusieurs clauses permettant de garantir l'uniformité et la qualité des services ${ }^{6} »$.

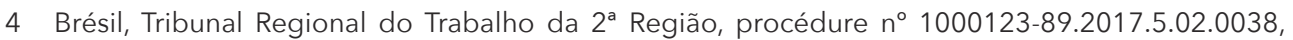
p. 3.

5 Brésil, Tribunal Regional do Trabalho da $2^{a}$ Região, procédure n 1000123-89.2017.5.02.0038, p. 7.

6 Brésil, Tribunal Regional do Trabalho da $2^{\text {a }}$ Região, procédure n 1000123-89.2017.5.02.0038, 1001574-25.2016.5.02.0026. 
Dans ces décisions, le modèle d'organisation de la plateforme d'Uber sert également de référence argumentative, d'une part sur la qualification de simple plateforme technologique, d'autre part sur le refus de la qualification d'employeur, car la spécificité de la composante technologique supprime la notion de la présence réelle d'un opérateur dans une relation de travail subordonnée.

\section{III - LE MODÈLE INTERPRÉTATIF DE LA JURISPRUDENCE}

Le principal critère prétorien utilisé pour décider de l'existence d'un travail subordonné repose toujours sur la délimitation juridique de la subordination, qui est établie par l'existence de contrôles et de sujétions. Le droit du travail brésilien (Consolidação das Leis do Trabalho - de 1943) définit le salarié comme «toute personne qui fournit des services de nature non contingente à un employeur, sous sa dépendance et moyennant un salaire » $\left(\right.$ art. $\left.3^{\circ}\right)$. C'est ainsi que, pour statuer sur l'existence d'une relation salariale entre les prestataires de service et l'entreprise qui les met en relation avec des clients, les juridictions nationales étudient l'existence factuelle de ces éléments classiques afin de déterminer le caractère subordonné de la relation de travail. La jurisprudence fait également dépendre la qualification de salarié, du caractère onéreux, régulier et personnel de la prestation, mais identifie en revanche l'employeur comme preneur de risque.

En dépit du fait que les décisions judiciaires ici commentées indiquent une tendance à procéder à des enquêtes factuelles sur les conditions de travail et sur le modèle organisationnel d'Uber, elles ont néanmoins souvent recours aux critères classiques de la subordination juridique. Si certaines décisions ont mis en évidence l'innovation technologique et le caractère perturbateur d'Uber, les éléments de subordination découlant des particularités du travail sur les plateformes numériques n'ont pas été pris en compte dans la définition juridique du lien de subordination. Pour attester de la présence d'indices classiques prouvant un lien de subordination, les décisions reconnaissant le statut de salarié ont avancé qu'Uber donnait des consignes sur les services à fournir. Dans ces affaires, les tribunaux ont estimé que, même si le chauffeur pouvait choisir quand et comment effectuer ces services, cela ne suffisait pas à exclure le lien de subordination. Le pouvoir de direction découle également de la possibilité de sanctionner le chauffeur, notamment en l'excluant de la plateforme.

La jurisprudence brésilienne reste incertaine sur la qualification juridique des services fournis par le biais de plateformes numériques. D'un point de vue plus théorique, le principal débat qui se déroule devant les prétoires est de savoir si cette nouvelle forme de travail doit être jugée à l'aune du critère classique du lien de subordination. Force est de constater que l'on a peu commenté l'insuffisance des critères de temps, d'espace et de délégation de travail pour identifier le lien de subordination dans ces nouvelles méthodes de production et de travail. La jurisprudence n'évoque donc pas l'hypothèse d'une inadéquation du modèle normatif actuel pour analyser les nouvelles formes de travail découlant de l'utilisation intensive des plateformes numériques. Dans le contenu intrinsèque des décisions de justice, aucun effort ne semble réellement avoir été entrepris pour élaborer de nouveaux critères de subordination, lesquels pourraient éventuellement mettre en lumière un déficit d'autonomie. Le chauffeur n'a pas accès aux informations principales, dissimulées par la plateforme, qui lui auraient pourtant permis, par exemple, de savoir où le passager souhaite se rendre. On peut dès lors en conclure qu'il n'est pas libre d'accepter ou de décliner une course. Certes, les refus sont possibles, mais ils restent limités et soumis à sanction, y compris la radiation du chauffeur de l'application. En outre, 


\section{BRÉSIL}

l'absence de coopération entre Uber et ses chauffeurs n'est pas non plus évoquée, car seul Uber contrôle les informations clés nécessaires à l'activité et peut les modifier de manière unilatérale. De plus, ces travailleurs indépendants ne voient aucune possibilité réelle de développer leur activité avec l'entreprise.

\section{CONCLUSION}

L'analyse des décisions de justice révèle de nouvelles tendances de la jurisprudence brésilienne, notamment en ce qui concerne la structure argumentative des arrêts. Toutefois, les jugements analysés, qui devaient évaluer la qualification juridique de ces nouveaux contrats, restent tributaires des indices classiques de la subordination juridique, ce qui révèle ainsi les efforts déployés pour faire entrer ces relations de travail dans le cadre institutionnel déjà existant. Malgré la volonté prétorienne de faire prévaloir la réalité factuelle sur les stipulations conventionnelles et la reconnaissance de nouvelles formes de travail, la jurisprudence ne semble pas encore admettre pleinement l'évolution des schémas classiques de dépendance et de subordination. 